Am J Ophthalmol. 2014 February ; 157(2): 479-487.e2. doi:10.1016/j.ajo.2013.09.027.

\title{
Lateral Rectus Superior Compartment Palsy
}

\author{
Robert A. Clark and Joseph L. Demer \\ Departments of Ophthalmology (R.A.C., J.L.D.) and Neurology (J.L.D.); Neuroscience (J.L.D.) \\ and Biomedical Engineering Interdepartmental Programs (J.L.D.); and David Geffen Medical \\ School (R.A.C., J.L.D.), University of California, Los Angeles, California
}

\section{Abstract}

PURPOSE-To employ magnetic resonance imaging (MRI) to seek evidence of compartmental lateral rectus atrophy consistent with a lesion involving selective denervation of only 1 of the 2 neuromuscular compartments of the lateral rectus.

DESIGN-Prospective observational case-control series.

METHODS-At a single institution, surface coil coronal MRI was obtained at $312 \mathrm{~mm}$ resolution in quasi-coronal planes $2 \mathrm{~mm}$ thick throughout the orbit in 20 normal volunteers and 18 patients with unilateral lateral rectus palsy who fixated monocularly on a target placed in central gaze. Maximum cross sections and posterior volumes of the superior and inferior lateral rectus compartments were computed and correlated with clinical findings.

RESULTS-Twelve patients with lateral rectus palsy demonstrated symmetric, highly significant $40 \%$ reductions in maximum cross sections and $50 \%$ reductions in posterior volumes from normal for both compartments ( $\mathrm{P}<10^{-6}$ for all comparisons). Six patients with lateral rectus palsy had similar significant but asymmetric reductions in those measures only for the superior compartment of the affected lateral rectus ( $\mathrm{P}<10^{-4}$ for all comparisons), with insignificant 20\%-30\% reductions for the inferior compartment $(\mathrm{P}>0.2$ for all comparisons).

CONCLUSIONS-A subset of patients with clinical lateral rectus palsy may have palsy limited to the superior compartment. Paralytic esotropia may be caused by lateral rectus superior compartment palsy despite an intact lateral rectus inferior compartment. This finding is consistent with evidence supporting independent innervation of the 2 lateral rectus neuromuscular compartments.

Histologic reconstruction demonstrates compartmental segregation of intramuscular motor innervation of mammalian horizontal rectus extraocular muscles, including those of humans. ${ }^{1,2}$ Prior to entering their target muscles, both the abducens nerve and oculomotor nerve divide into superior and inferior branches that innervate minimally overlapping

(C) 2014 Published by Elsevier Ltd.

Inquiries to Joseph L. Demer, Jules Stein Eye Institute, 100 Stein Plaza, UCLA, Los Angeles, CA 90095-7002; jld@ucla.edu. ALL AUTHORS HAVE COMPLETED AND SUBMITTED THE ICMJE FORM FOR DISCLOSURE OF POTENTIAL CONFLICTS OF INTEREST. None of the authors have any financial disclosures to report. The authors have no proprietary interests in the surface coils.

Contributions of authors: design of the study (R.C., J.D.); conduct of the study (R.C., J.D.); collection, management, analysis, and interpretation of the data (R.C., J.D.); and preparation, review, and approval of the manuscript (R.C., J.D.). 
neuromuscular compartments that remain vertically segregated over the entire anteroposterior length of each muscle. ${ }^{1,2}$ Differential innervation of the horizontal rectus muscle compartments appears to contribute to ocular counter-rolling. ${ }^{3}$ Since there is substantial mechanical independence of the parallel muscle and tendon fibers, ${ }^{4}$ the broad insertions allow the superior and inferior compartments to exert forces displaced sufficiently from the horizontal globe meridian to generate substantial torsional and vertical torques, in much the same manner as surgical transposition of the tendon insertion perpendicular to its path imparts cyclovertical action to the transposed extraocular muscle. ${ }^{3,5-7}$

Segregated innervation of horizontal rectus muscles suggests the potential existence of a differential response to pathologic injury of nerve branches innervating the 2 functional compartments. Despite proximity of vertical neuromuscular compartments, a compressive lesion, demyelinating disease, microvascular insult, trauma, or other pathology of the abducens or oculomotor nerve $\mathrm{e}^{8-10}$ might selectively or differentially denervate only 1 compartment, sparing the other. For the lateral rectus muscle in particular, historically considered to mediate only abduction, selective compartmental palsy might introduce vertical and/or torsional deficits in addition to the classic paralytic esotropia, findings that could confuse the clinical presentation 11,12 and invite the erroneous diagnosis of a concurrent cyclovertical muscle palsy or skew deviation. Indeed, cyclovertical deviations have been reported frequently to distort the Listing's law relationship to ocular torsion in "central" forms of lateral rectus palsy. ${ }^{12}$ This interpretation, however, and the belief that loss of fusion could create vertical and excyclotorsional misalignments in isolated lateral rectus palsy, preceded recognition of compartmental innervation of extraocular muscles.

High-resolution magnetic resonance imaging (MRI) has been used in prior studies of extraocular muscle contractility, both in normal subjects ${ }^{13-15}$ and in subjects with cranial nerve palsies. ${ }^{16-20}$ These studies consistently demonstrate an increase in maximum crosssectional area, a shift of the image plane of maximum cross section posteriorly, and an increase in posterior extraocular muscle volume during contraction, changes in functional anatomy diminished or ablated by palsy of the innervating cranial nerve. Using careful technique, MRI resolution is sufficient to detect compartmental changes in extraocular muscle morphology corresponding to contractile state, ${ }^{3}$ and thus has the potential to detect partial paresis of the extraocular muscle belly differentially affecting distinct neuromuscular compartments. This study used MRI to seek evidence of asymmetric compartmental atrophy in patients with clinical lateral rectus palsy.

\section{METHODS}

This study was conducted at a single institution as a prospective observational case-control series. Participants gave written informed consent to a protocol conforming to the Declaration of Helsinki and approved by the Institutional Review Board at the University of California, Los Angeles. All data collection was Health Insurance Portability and Accountability Act compliant. Twenty normal, orthotropic paid volunteer adults were recruited by advertisement. Each control underwent comprehensive eye examination to verify normal acuity, ocular motility, stereoacuity, and ocular anatomy. From 1996 to 2012, 18 patients with unilateral chronic lateral rectus palsy were recruited prospectively through 
an ongoing study. Each patient with strabismus underwent a similar examination. Ductions were scaled from 0 (normal) to -4 (just able to reach central gaze from adduction). Fundus torsion was determined either using the double Maddox rod or by objectively determining the angle between the fovea and the center of the optic disc, ${ }^{21,22}$ as measured with a +90 refractive diopter condensing lens at the slit lamp as the patient foveated a rotating slit beam whose angle was adjusted until the beam intercepted both the fovea and the center of the optic disc. ${ }^{23}$

High-resolution T1- or T2-weighted fast spin echo MRI was performed on each participant with a 1.5-T General Electric Signa (Milwaukee, Wisconsin, USA) scanner using techniques detailed elsewhere, ${ }^{3,24,25}$ including use of a dual-phased surface coil array (Medical Advances, Milwaukee, Wisconsin, USA) to improve signal-to-noise ratio and a fixation target to avoid motion artifacts. Magnetic resonance imaging surface coils used in this project were not approved by the US Food and Drug Administration for this purpose. Participants were scanned supine with the head stabilized. During imaging, subjects fixated a fine optical fiber $2 \mathrm{~cm}$ distant and illuminated from its distal end by a red light-emitting diode. The fiber was positioned to keep the scanned eye as close to central gaze as possible.

Initially, low-resolution craniotopic images of both orbits were obtained simultaneously at 3 $\mathrm{mm}$ thickness with $780 \mu \mathrm{m}$ in-plane resolution. Low-resolution images were used to place sets of 18-20 contiguous, 2-mm-thick quasi-coronal images in a plane perpendicular to the long axis of each orbit using a $256 \times 256$ matrix over an $8-\mathrm{cm}$ field of view, yielding $312 \mu \mathrm{m}$ in-plane resolution.

Digital MRI images were quantified using both the program ImageJ64 (W. Rasband, National Institutes of Health, Bethesda, Maryland, USA; http://rsb.info.nih.gov/ij/, 19972009) and custom image analysis programs developed using the MatLab platform (MathWorks, Boston, Massachusetts, USA; 2011) employing techniques described in detail elsewhere. ${ }^{3}$ In summary, beginning as far posteriorly in each orbit as the lateral rectus belly could be distinguished, each lateral rectus was manually outlined and saved as a file that included only the lateral rectus belly. The lateral rectus image was then processed in MatLab by first rotating the entire image to align the vertical axis of the muscle with scanner vertical (Figure 1, Left), and then calculating the areas of the superior and inferior lateral rectus compartments by separately counting the pixels in the superior $40 \%$ and inferior $40 \%$ of the vertical extent of the muscle (Figure 1, Right), neglecting the middle $20 \%$ of the muscle with potential intermingled innervation. ${ }^{1,2}$

Cross-sectional areas were measured for the superior and inferior compartments in all image planes. Then, the compartmental areas of the 4 contiguous image planes from 8-14 mm posterior to the globe-optic nerve junction, the most posterior image planes that were available in every subject, were multiplied by the $2-\mathrm{mm}$ slice thickness and summed to compute the posterior partial volume for each lateral rectus compartment. ${ }^{15}$ Two morphometric measures were analyzed: the single-plane maximum cross-sectional area for the mid-orbital region (6-12 mm posterior to the globe) and the posterior partial volume. ${ }^{15}$ The superior and inferior compartmental areas and posterior partial volumes were then scaled as fractions of those measures for each entire muscle. For each measure, the relative 
proportion of the superior compartment was plotted against the relative proportion in the inferior compartment to assess possible clustering that would indicate asymmetric compartmental lateral rectus atrophy.

\section{RESULTS}

Quasi-coronal MRI provided detailed lateral rectus cross sections throughout the orbit and, as expected, confirmed reduced size of the lateral rectus belly in all 18 patients with lateral rectus palsy. The patients clearly segregated into 2 groups based exclusively on the differences in the 2 morphometric measures. Figure 2 illustrates MRI of the entire orbit contrasting palsied and unaffected lateral rectus muscles of 2 representative patients. In 12 cases, the entire lateral rectus cross section was uniformly atrophic, as illustrated for the right orbit image in Figure 2 for Patient 1, and for cropped lateral rectus images in all 12 cases in Figure 3. These 12 patients were considered to have complete lateral rectus palsies. As shown in Table 1, the superior and inferior compartments of the paretic lateral rectus exhibited an average decrease in size nearly identical for both maximum cross-sectional area (42.1\% vs $42.9 \%)$ and posterior partial volume (49.3\% vs $46.9 \%)$.

The other 6 patients with lateral rectus palsy exhibited asymmetric atrophy mainly affecting the superior compartment, but sparing the inferior compartment, of the palsied lateral rectus. This asymmetric atrophy is illustrated in the right orbit image in Figure 2 for Patient 16 and for cropped lateral rectus images in all 6 cases in Figure 4. In each case, obvious relative preservation of the inferior portion of the palsied lateral rectus is interpreted as evidence of selective superior compartment atrophy. Despite individual variations in morphology and size for both normal and involved lateral rectus, in each case, the asymmetric atrophy of the superior lateral rectus belly is apparent, as is relative inferior compartment sparing. As shown in Table 2, the superior compartment had twice the loss of maximum cross-sectional area $(46.5 \%)$ as the inferior compartment $(23.4 \%)$, with nearly twice the difference in posterior volume (53.8\% vs $31.6 \%)$.

Two-dimensional graphical analysis demonstrated that all 6 lateral rectus muscles with superior compartment palsy differed morphometrically from both normal and completely palsied lateral rectus muscles. This analysis involved plotting separately for maximum cross-sectional area (Figure 5, Left) and posterior partial volume (Figure 5, Right) on the abscissa the proportion (relative to the total for the muscle) in the inferior lateral rectus compartment and on the ordinate the proportion in the superior compartment. For each of these 2 measures of size, both control and completely palsied lateral rectus muscles exhibited similar proportions in the inferior and superior lateral rectus compartments, so that data points for each muscle clustered in the upper left of each plot (Figure 5). For lateral rectus muscles affected asymmetrically, however, the muscle proportion in the inferior compartment was relatively large and the proportion in the superior compartment was relatively small, so the data points clustered in the lower right portion of each graph in the region demarcated by the gray borders. Clustering of cross-sectional area (Figure 5, Left) and posterior partial volume (Figure 5, Right) data is similar, supporting the validity of this approach. This unique morphologic clustering of lateral rectus muscles with superior compartment atrophy is interpreted as distinguishing this pathology from complete lateral 
rectus palsy. The clustering also defines natural break points in the data for classifying patients as having complete vs compartmental lateral rectus palsies.

The paretic lateral rectus superior compartments of patients with both superior compartment and complete lateral rectus palsy had highly statistically significantly smaller maximum cross-sectional areas and posterior volumes than controls $\left(P<10^{-4}\right.$ for all comparisons). For complete lateral rectus palsy, the inferior lateral rectus compartment was also highly significantly subnormal $\left(P<10^{-6}\right.$ for both maximum cross section and posterior partial volume). However, the 6 patients with superior compartment palsies had inferior compartment maximum cross sections and posterior partial volumes indistinguishable from normal $(P>.2$ for both measures).

Clinical characteristics and motility features of patients with lateral rectus palsies are compared in Table 3.A neurologic etiology was identified for the palsy in all but Patients 7 and 15. Patients 3, 5, 6, 10, 11, and 13 had concurrent vertical muscle palsies. Prior to MRI, Patient 4 underwent strabismus surgery consisting of right medial rectus recession with half tendon width inferior transposition, right lateral rectus resection with half tendon superior transposition, and anterior transposition of the anterior half of the right superior oblique tendon. Patient 8 had Duane retraction syndrome type 1 prior to developing ipsilateral lateral rectus paralysis owing to meningioma. Patient 13 underwent 4 strabismus surgeries prior to MRI: (1) right inferior rectus recession; (2) right inferior rectus advancement; (3) right medial rectus recession/right lateral rectus resection; and (4) bilateral medial rectus and left inferior rectus recession. None of the surgeries involved the paretic left lateral rectus. Patient 16 underwent 2 strabismus surgeries prior to MRI: (1) right medial rectus recession/right lateral rectus resection; and (2) left medial rectus recession/right lateral rectus plication.

Patients with complete lateral rectus palsy had a larger average central gaze esotropia, $44.8 \Delta$, compared with $31.7 \Delta$ for patients with superior compartment palsies, but the difference was not statistically significant $(P=.24)$. Patients with superior compartment palsy had significantly greater abduction, graded at -2.5 , than did patients with complete lateral rectus palsy at $-4.0(P=.03$, Mann-Whitney $U$ 2-tailed test). Insufficient torsional and vertical data were available for statistical comparison.

\section{DISCUSSION}

This study employed high-resolution MRI to identify a subgroup of patients with lateral rectus weakness associated with selective atrophy of the superior more than inferior compartment of the lateral rectus. Neurogenic atrophy of extraocular muscles has been shown to occur reliably and rapidly following denervation. ${ }^{16-20,26}$ The finding of selective atrophy of the lateral rectus superior compartment represents a pathologic correlate to recent anatomic recognition that peripheral lateral rectus innervation has roughly equal superior and inferior compartments, ${ }^{1,2}$ and the demonstration by functional MRI of differential activation of the 2 compartments during ocular counter-rolling. ${ }^{3}$ It is proposed that patients with selective superior lateral rectus atrophy exhibit asymmetric lateral rectus superior compartment palsy, representing selective neurogenic atrophy. This condition is defined by weakness predominantly or exclusively of the superior lateral rectus compartment, with 
relative preservation of structure and function of the inferior lateral rectus compartment. Thus, some patients with clinical lateral rectus palsy exhibited relative preservation of inferior compartment morphology, presumably because of sparing of the inferior branch of the abducens nerve. These patients, on average, exhibited significantly greater abduction than those with palsy of both lateral rectus compartments, as might be anticipated from preservation of abducting function in the inferior lateral rectus compartment. Patients with superior compartment lateral rectus palsy often exhibited concurrent excyclotorsion in the absence of cyclovertical muscle dysfunction. Both hypotropia and excyclotorsion could result from application of lateral rectus inferior compartment force to the sclera inferior to the globe's horizontal meridian, in the absence of counterbalancing force owing to paralysis of the lateral rectus superior compartment. ${ }^{3}$

It is curious that all incomplete lateral rectus palsies involved superior compartment atrophy. The likelihood of all 6 asymmetric palsies randomly involving only the superior compartment is $1.6 \%$ (binomial distribution probability of $1 / 2$ raised to the 6th power), assuming equal chance of superior and inferior compartment involvement. It is unknown which features of the superior division of the abducens nerve might leave it more susceptible to injury, but a similar observation has been made in Duane syndrome, where the lateral rectus inferior compartment is more likely to be spared atrophy when anomalously innervated by the oculomotor nerve. ${ }^{27}$ Given that the atrophy respected the anatomic borders of the abducens nerve branches, plus given the homogenous nature of the muscle fibers and known neurologic deficits in all but 1 case, the asymmetric atrophy observed on MRI is not likely attributable to some unknown factor such as a new type of muscular dystrophy or other intrinsic muscular disorder. However, an unknown alternative cause of selective atrophy cannot be excluded.

Selective compartmental lateral rectus atrophy could alter the effects of typical strabismus surgeries used to treat lateral rectus palsy. Clinically, severity of duction deficit and other signs of residual lateral rectus function are used to plan surgery. ${ }^{28,29}$ If residual lateral rectus abducting force exists, manifested by abduction beyond the midline, the paretic lateral rectus is typically tightened by tendon resection combined with ipsilateral medial rectus recession. ${ }^{29,30}$ The present findings imply, however, that lateral rectus palsy with better abduction more likely represents predominantly superior compartment palsy. If the inferior compartment is relatively preserved, lateral rectus resection may induce or exacerbate vertical or torsional strabismus by asymmetrically increasing inferior compartment force without augmenting force in the atrophic superior compartment.

When a surgeon determines that minimal abducting force is generated by the paretic lateral rectus, temporal transposition of both vertical rectus muscles is usually performed, sometimes with augmentation through scleral posterior fixation sutures. ${ }^{28,29,31}$ Although in the present study larger duction deficits were associated with more symmetrical lateral rectus compartmental atrophy, 2 of the 6 patients with asymmetric compartmental atrophy, Patients 17 and 18, had abduction graded at -4 and therefore might be offered transposition surgery. For those patients, symmetric vertical muscle transpositions might induce or exacerbate vertical or torsional strabismus. ${ }^{28,32}$ Vertical strabismus has been observed to be problematic after some cases of vertical rectus transposition for abducens palsy. ${ }^{33}$ Selective 
preservation of lateral rectus inferior compartment function may explain why transposition of the ipsilateral superior rectus alone successfully treats lateral rectus palsy, ${ }^{34}$ without ipsilateral inferior rectus transposition.

Post hoc analysis of the clinical presentation suggests some indicators that should alert clinicians to the possibility of lateral rectus superior compartment palsy. Relative preservation of the inferior lateral rectus compartment was associated with significantly better abduction and a trend toward less central gaze esotropia than with complete lateral rectus palsy. Ipsilateral hypotropia, in either central gaze or attempted abduction, and excylotorsion are also suggestive. Most patients with lateral rectus palsy in both groups (14 out of 18) had compressive or traumatic etiologies, possibly reflecting institution-based recruitment, ${ }^{10}$ so etiology was unhelpful in distinguishing compartmental from complete palsy. Possible coexistence of concurrent vertical rectus pathology, however, necessitates clinical orbital imaging to confirm the diagnosis. Fortunately, large asymmetry in maximum cross-sectional area between compartments is obvious upon visual inspection of lateral rectus morphology, making it clinically unnecessary to perform quantitative measurements of cross-sectional area or posterior volume.

Neuromuscular compartmentalization is present in the medial rectus, but not in the vertical rectus extraocular muscles. ${ }^{2}$ Isolated medial rectus compartmental palsy could theoretically create a similar clinical presentation, but since medial rectus palsy typically occurs in the context of oculomotor nerve palsy that also denervates the vertical rectus muscles, the added effects of asymmetric medial rectus atrophy on the vertical and torsional components of the strabismus would be difficult to recognize or quantify.

Strengths of the present study include the use of high-resolution MRI, automated segmentation of lateral rectus images for quantitative analysis of lateral rectus morphology, and inclusion of a relatively large number of prospectively recruited controls and patients with lateral rectus palsy. Only 6 patients with lateral rectus superior compartment palsy, however, were identified for detailed study. Moreover, during the long time interval during which patients with lateral rectus compartmental palsy were ascertained, existence of this novel clinical entity was only gradually suspected. Associated clinical features of cyclovertical strabismus augmenting lateral rectus palsy were not recognized until relatively late in the ascertainment for this study, so clinical investigations of heterotropia in early patients were sparse, a limitation understandable in the setting of large-angle paralytic esotropia. It should be possible to better characterize the clinical features of lateral rectus superior compartment palsy in the future, now that the existence of the disorder is recognized. It is likely that this condition exists in a substantial minority of all cases of lateral rectus palsy, but orbital imaging is necessary for confirmation.

In conclusion, some patients with lateral rectus palsy may have selective palsy of only the lateral rectus superior compartment. Paralytic esotropia may be caused by isolated lateral rectus superior compartment palsy with relative preservation of the lateral rectus inferior compartment, and the asymmetric atrophy may complicate the clinical presentation and the possible response to standard strabismus surgeries. Recognition of this pathophysiologic entity may clarify the diagnosis of paralytic esotropia. 


\section{Acknowledgments}

This study was supported by the U.S. Public Health Service (NEI grant EY08313), Research to Prevent Blindness, and The Shaw Family Endowment Fund.

\section{REFERENCES}

1. Peng M, Poukens V, da Silva Costa RM, Yoo L, Tyschen L, Demer JL. Compartmentalized innervation of primate lateral rectus muscle. Invest Ophthalmol Vis Sci. 2010; 51(9):4612-4617. [PubMed: 20435590]

2. da Silva Costa RM, Kung J, Poukens V, Yoo L, Tyschen L, Demer JL. Intramuscular innervation of primate extraocular muscles: unique compartmentalization in horizontal recti. Invest Ophthalmol Vis Sci. 2011; 52(5):2830-2836. [PubMed: 21220556]

3. Clark RA, Demer JL. Differential lateral rectus compartmental contraction during ocular counterrolling. Invest Ophthalmol Vis Sci. 2012; 53(6):2887-2896. [PubMed: 22427572]

4. Shin A, Yoo L, Chaudhuri Z, Demer JL. Independent passive mechanical behavior of bovine extraocular muscle compartments. Invest Ophthalmol Vis Sci. 2012; 53(13):8414-8423. [PubMed: 23188730]

5. von Noorden G, Chu MW. Surgical treatment options in cyclotropia. J Pediatr Ophthalmol Strabismus. 1990; 27(6):291-293. [PubMed: 2086744]

6. Ohmi G, Fujikado T, Ohji M, Saito Y, Tano Y. Horizontal transposition of vertical rectus muscles for treatment of excyclotropia. Graefes Arch Clin Exp Ophthalmol. 1997; 235(1):1-4. [PubMed: 9034834]

7. Kono R, Ohtsuki H, Okanobu H, Kingugasa K. Displacement of rectus muscle pulleys by torsional muscle surgery for treatment of full macular translocation-induced incyclotropia. Am J Ophthalmol. 2005; 140(1):144-146. [PubMed: 16038663]

8. Patel S, Holmes J, Hodge D, Burke J. Diabetes and hypertension in isolated sixth nerve palsy: a population-based study. Ophthalmology. 2005; 112(5):760-763. [PubMed: 15878054]

9. Richards B, Jones F, Younge B. Causes and prognosis in 4,278 cases of analysis of the oculomotor, trochlear, and abducens cranial nerves. Am J Ophthalmol. 1992; 113(5):489-496. [PubMed: 1575221]

10. Patel S, Mutyala S, Leske D, Hodge D, Holmes J. Incidence, associations, and evaluation of sixth nerve palsy using a population-based method. Ophthalmology. 2004; 111(2):369-375. [PubMed: 15019392]

11. Slavin M. Hyperdeviation associated with isolated unilateral abducens palsy. Ophthalmology. 1989; 96(4):512-516. [PubMed: 2726181]

12. Wong A, Tweed D, Sharpe J. Vertical misalignment in unilateral sixth nerve palsy. Ophthalmology. 2002; 109(7):1315-1325. [PubMed: 12093657]

13. Miller JM. Functional anatomy of normal human rectus muscles. Vision Res. 1989; 29(2):223240. [PubMed: 2800349]

14. Tian S, Nishida Y, Isberg B, Lennerstrand G. MRI measurements of normal extraocular muscles and other orbital structures. Graefes Arch Clin Exp Ophthalmol. 2000; 238(5):393-404. [PubMed: 10901470]

15. Clark RA, Demer JL. Functional morphometry of horizontal rectus extraocular muscles during ocular duction. Invest Ophthalmol Vis Sci. 2012; 53(11):7375-7379. [PubMed: 22997285]

16. Demer JL, Miller JM, Koo EY, Rosenbaum AL. Quantitative magnetic resonance morphometry of extraocular muscles: a new diagnostic tool in paralytic strabismus. J Pediatr Ophthalmol Strabismus. 1994; 31(3):177-188. [PubMed: 7931952]

17. Demer JL, Miller JM. Magnetic resonance imaging of the functional anatomy of the superior oblique muscle. Invest Ophthalmol Vis Sci. 1995; 36(5):906-913. [PubMed: 7706039]

18. Bloom JN, Cadera W, Heiberg E, Karlik S. A magnetic resonance imaging study of horizontal rectus muscle palsies. J Pediatr Ophthalmol Strabismus. 1993; 30(5):296-300. [PubMed: 8254444] 
19. Tsai TH, Demer JL. Nonaneurysmal cranial nerve compression as cause of neuropathic strabismus:evidence from high-resolution magnetic resonance imaging. Am J Ophthalmol. 2011; 152(6):1067-1073. [PubMed: 21861970]

20. Demer JL, Ortube MC, Engle EC, Thacker N. High-resolution magnetic resonance imaging demonstrates abnormalities of motor nerves and extraocular muscles in patients with neuropathic strabismus. J AAPOS. 2006; 10(2):135-142. [PubMed: 16678748]

21. Guyton D. Clinical assessment of ocular torsion. Am Orthoptic J. 1983; 33:7-15.

22. Bixenman W, von Noorden G. Apparent foveal displacement in normal subjects and in cyclotropia. Ophthalmology. 1982; 89(1):58-62. [PubMed: 7070775]

23. Kothari M, Venkatesan G, Shah J, Kothari K, Nirmalan P. Can ocular torsion be measured using the slit lamp biomicroscope? Indian J Ophthalmol. 2005; 53(1):43-47. [PubMed: 15829746]

24. Demer JL, Clark RA. Magnetic resonance imaging of human extraocular muscles during static ocular counter-rolling. J Neurophysiol. 2005; 94(5):3292-3302. [PubMed: 16033934]

25. Demer JL, Dusyanth A. T2-weighted fast spin-echo magnetic resonance imaging of extraocular muscles. J AAPOS. 2011; 15(1):17-23. [PubMed: 21397801]

26. Demer JL, Poukens V, Ying H, Shan X, Tian J, Zee DS. Effects of intracranial trochlear neurectomy on the structure of the primate superior oblique muscle. Invest Ophthalmol Vis Sci. 2010; 51(7):3485-3493. [PubMed: 20164458]

27. Demer JL, Clark RA, Lim KH, Engle EC. Magnetic resonance imaging evidence for widespread orbital dysinnervation in dominant Duane's retraction syndrome linked to the DURS2 locus. Invest Ophthalmol Vis Sci. 2007; 48(1):194-202. [PubMed: 17197533]

28. Rosenbaum AL. The efficacy of rectus muscle transposition surgery in esotropic Duane syndrome and VI nerve palsy. J AAPOS. 2004; 8(5):409-419. [PubMed: 15492732]

29. Rosenbaum, AL.; Santiago, AP. Clinical Strabismus Management: Principles and Surgical Techniques. Philadelphia: W.B. Saunders Company; 1999. p. 552-555.

30. Altintas AG, Arifoglu HB, Dal D, Simsek S. Are most sixth nerve palsies really paralytic? J Pediatr Ophthalmol Strabismus. 2011; 48(3):187-191. [PubMed: 20672767]

31. Foster RS. Vertical muscle transposition augmented with lateral fixation. J AAPOS. 1996; 1(1): 20-30. [PubMed: 10530981]

32. Holmes J, Hatt SR, Leske D. Intraoperative monitoring of torsion to prevent vertical deviations during augmented vertical rectus transposition surgery. J AAPOS. 2012; 16(2):136-140. [PubMed: 22525168]

33. Ruth AL, Velez FG, Rosenbaum AL. Management of vertical deviations after vertical rectus transposition surgery. J AAPOS. 2009; 13(1):16-19. [PubMed: 19117778]

34. Mehendale RA, Dagi LR, Wu C, Ledoux D, Johnston S, Hunter DG. Superior rectus transposition and medial rectus recession for Duane syndrome and sixth nerve palsy. Arch Ophthalmol. 2012; 130(2):195-201. [PubMed: 22332212]

\section{Biographies}

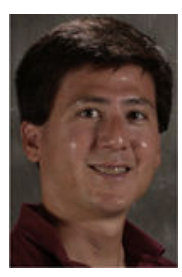

Robert A. Clark, MD, has been on the clinical faculty at the Jules Stein Institute for more than 16 year. He has more than fifty publications, written a book on children's eyecare for parents, designed new surgical instruments for pediatric surgery, and created new strabismus surgery techniques. He received an Honor Award for his contributions to the American 
Association for Pediatric Ophthalmology \& Strabismus and is on the editorial board of the Journal of AAPOS.

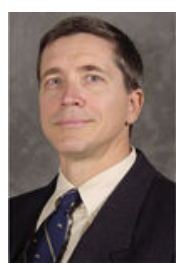

Joseph L. Demer, MD, PhD is Leonard Apt Professor, Chief of Pediatric Ophthalmology and Strabismus, and Professor of Neurology, at the David Geffen School of Medicine, University of California Los Angeles. Dr Demer received the Friedenwald Award from the Association for Research in Vision and Ophthalmology, and received a Recognition Award from the Alcon Research Institute, for his work on the extraocular muscles and orbital connective tissues. 

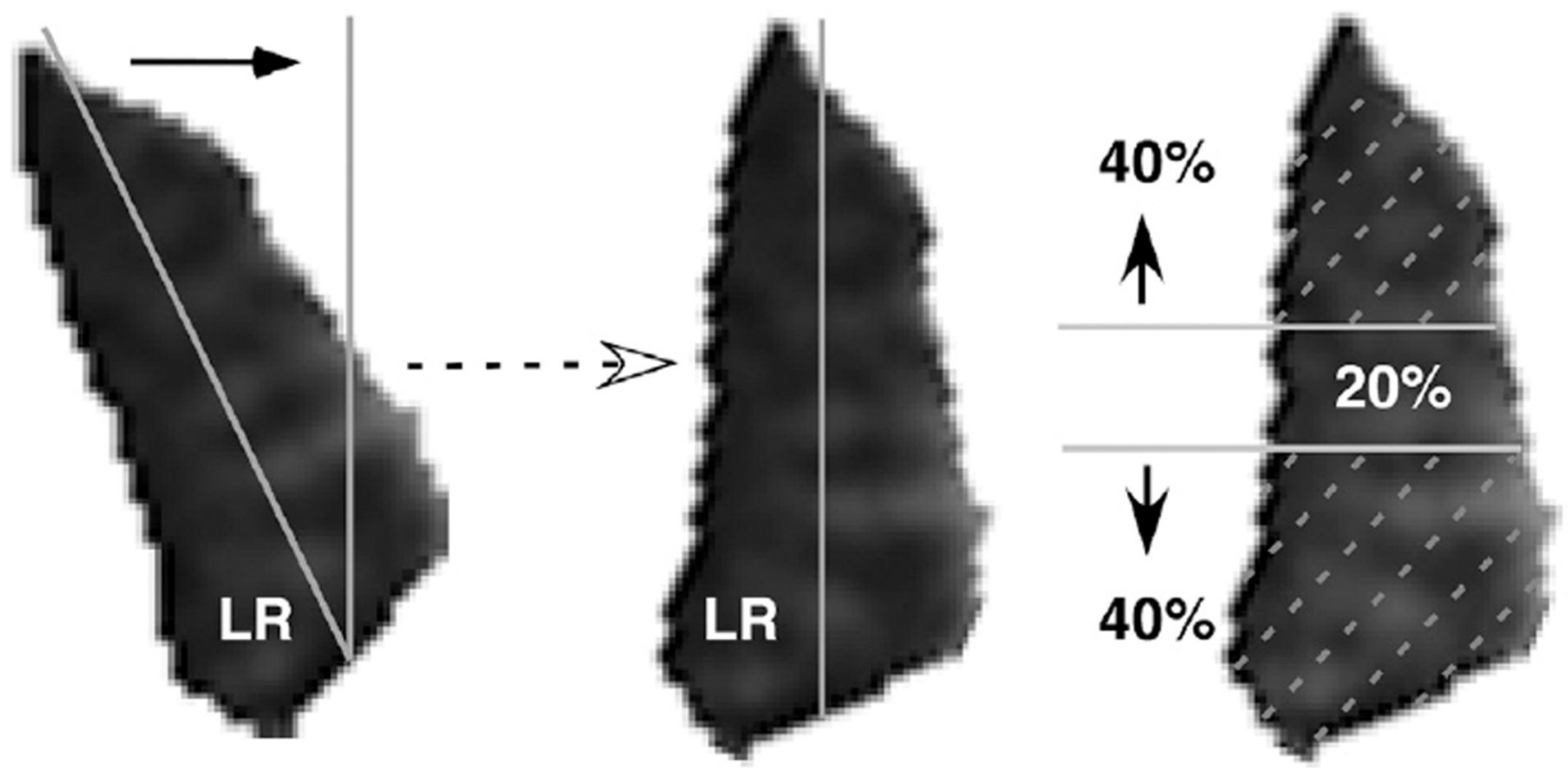

FIGURE 1.

Lateral rectus cross section. A computer program automatically calculated morphology of each cross section of the lateral rectus (LR) muscle belly. (Left) A line was best fit along the greatest dimension, and the image was then rotated to align this line with scanner vertical. (Right) The area was computed within the superior $40 \%$ and inferior $40 \%$ of the vertical extent of each rotated cross section, excluding the middle $20 \%$ because this region might contain mixed innervation from both the superior and inferior abducens nerve branches. 


\section{Patient 1}

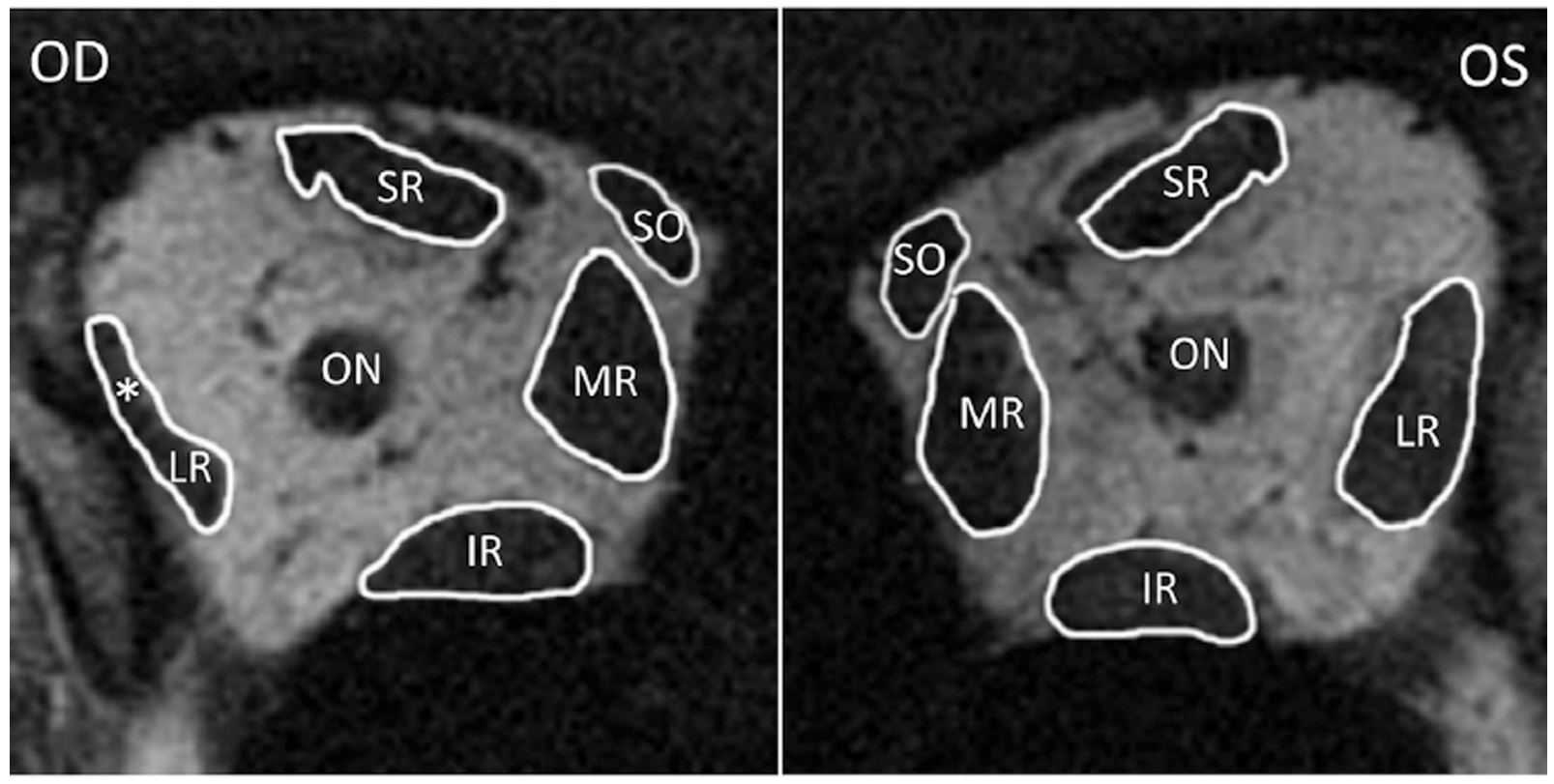

Patient 16
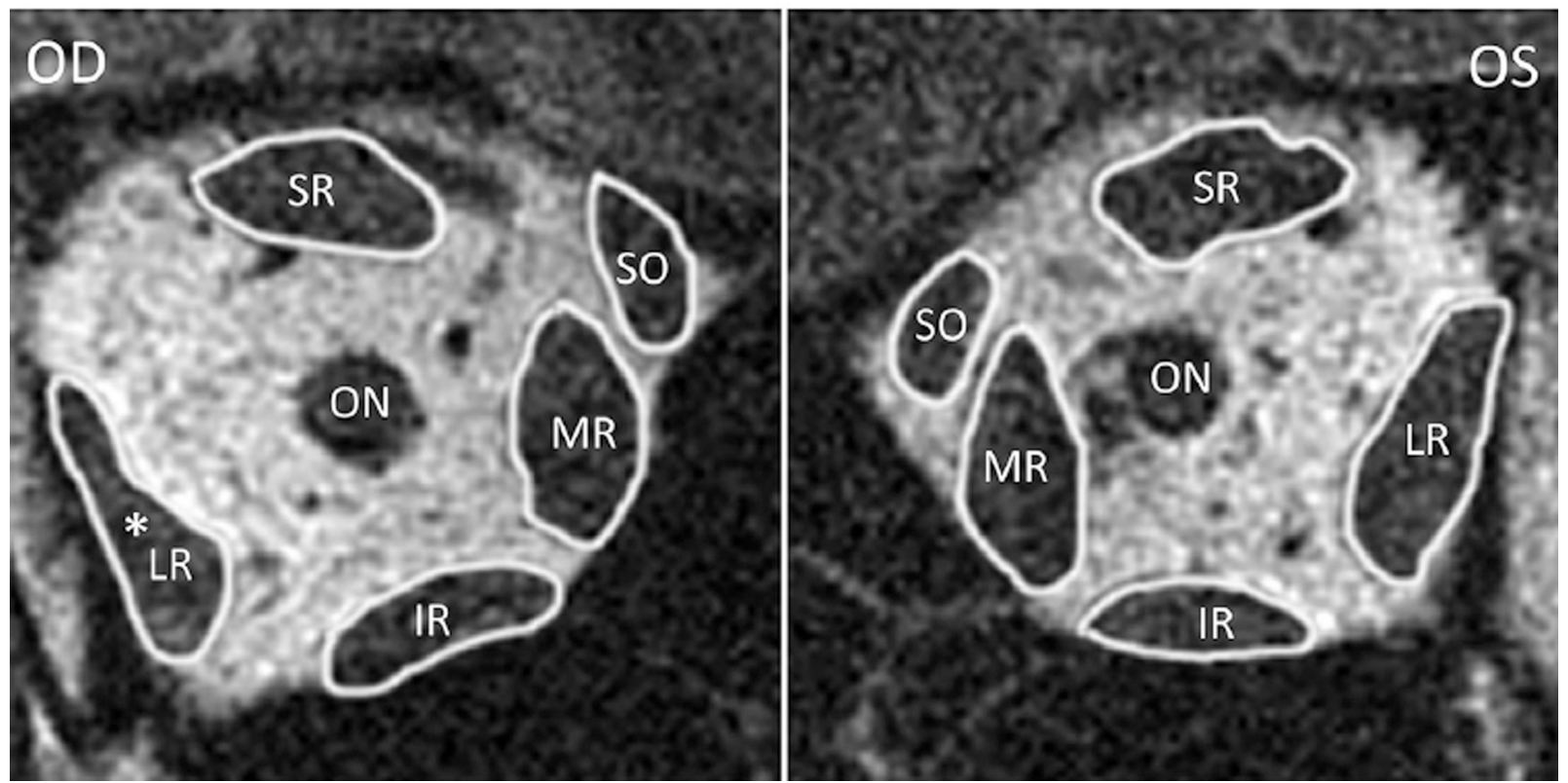

FIGURE 2.

Quasi-coronal magnetic resonance images of normal and paretic (white asterisk) orbits. Patient 1 has a right lateral rectus complete palsy and Patient 16 has a right lateral rectus superior compartment palsy. In both patients, the paretic lateral rectus was obviously smaller than the uninvolved lateral rectus. $\mathrm{SR}=$ superior rectus muscle; $\mathrm{SO}=$ superior oblique muscle; $\mathrm{MR}=$ medial rectus muscle; $\mathrm{IR}=$ inferior rectus muscle; $\mathrm{LR}=$ lateral rectus; $\mathrm{OD}=$ right eye; $\mathrm{ON}=$ optic nerve; $\mathrm{OS}=$ left eye. 

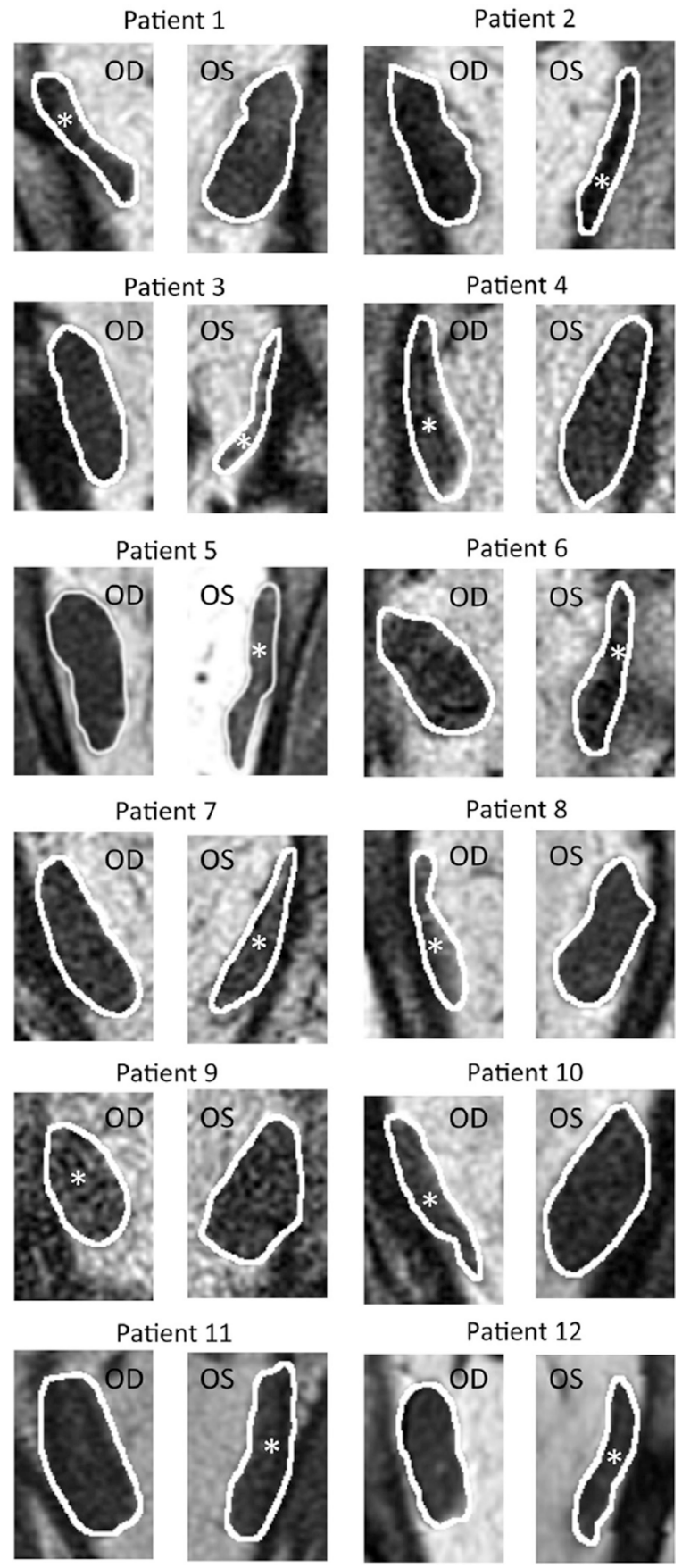

FIGURE 3.

Magnetic resonance images of normal and completely paretic (white asterisk) lateral rectus muscles. All patients shown have unilateral complete lateral rectus atrophy. $\mathrm{OD}=$ right eye; $\mathrm{OS}=$ left eye. 
Patient 13
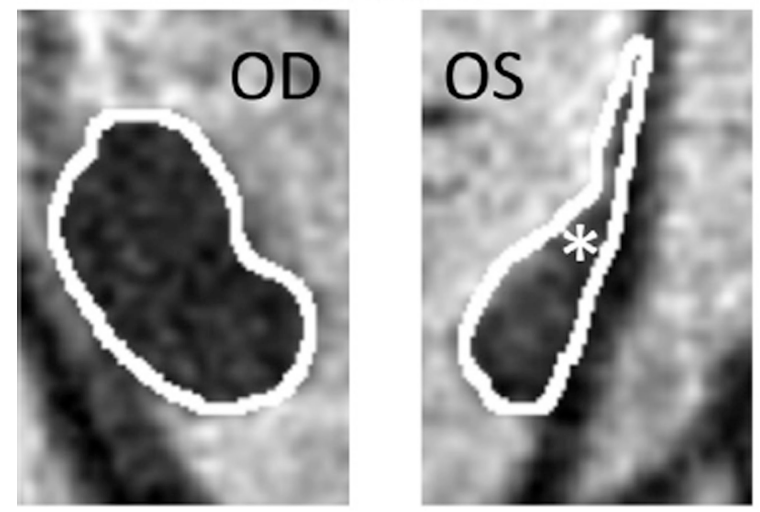

Patient 15
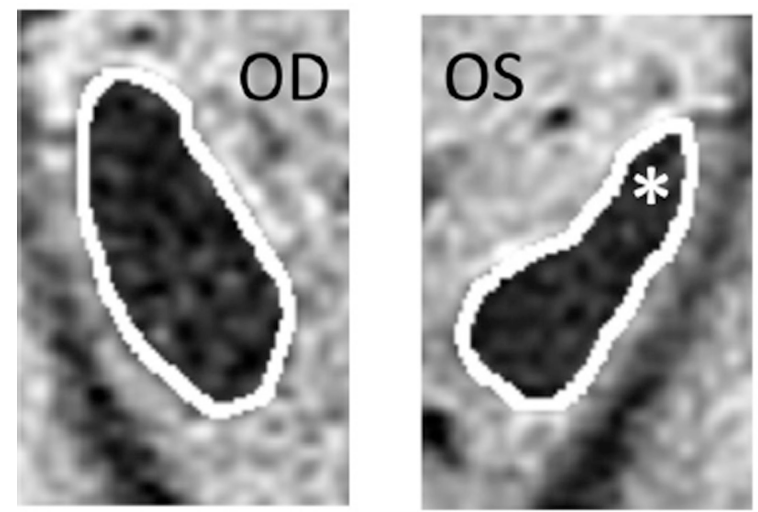

\section{Patient 17}
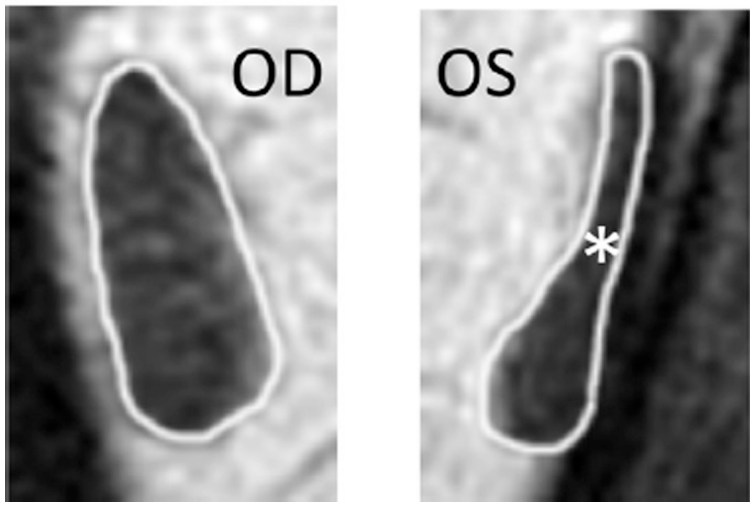

FIGURE 4.

Magnetic resonance images of normal and partially paretic (white asterisk) lateral rectus muscles. All patients shown have unilateral lateral rectus superior compartment atrophy. $\mathrm{OD}=$ right eye; $\mathrm{OS}=$ left eye. 

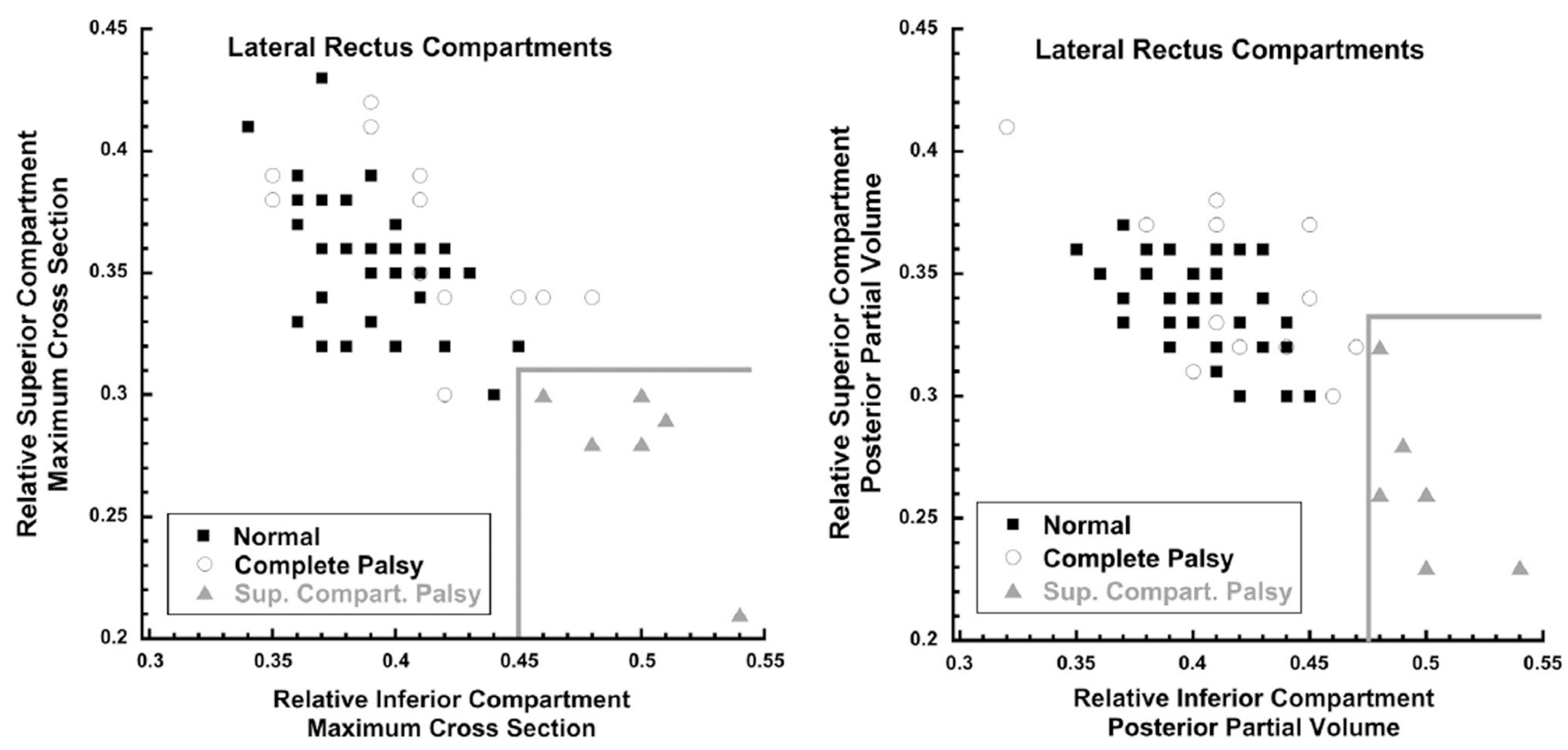

FIGURE 5.

Relative proportion of lateral rectus within superior vs inferior compartment. (Left) Maximum cross-sectional area. Controls and patients with complete lateral rectus palsy all had a less than 0.14 (14\%) difference between superior vs inferior maximum cross sections, so data points for these muscles cluster to the upper left. The 6 affected muscles with superior compartment palsy (gray triangles) had inferior compartment maximum cross sections at least $0.16(16 \%)$ greater than the inferior compartment maximum cross sections, so these data points clustered toward the lower right in the region demarcated by the gray lines.

(Right) Posterior partial volume. Controls and patients with symmetric lateral rectus palsy all had less than 0.16 (16\%) difference between the superior and inferior compartments. Six superior compartment lateral rectus palsies (gray triangles) had inferior compartment posterior partial volumes at least $0.16(16 \%)$ greater than inferior compartment values, so that their data points clustered toward the bottom right of the graph in the region demarcated by the gray lines. 


\section{TABLE 1}

\section{Lateral Rectus Size in Complete Palsy}

\begin{tabular}{cccccc}
\hline & \multicolumn{2}{c}{ Maximum Cross Section $\left(\mathbf{m m}^{\mathbf{2}}\right)$} & & \multicolumn{2}{c}{ Posterior Volume $\left(\mathbf{m m}^{\mathbf{3}}\right)$} \\
\cline { 2 - 3 } \cline { 5 - 6 } Patient & Sup & Inf & & Sup & Inf \\
\cline { 5 - 6 } 1 & Compartment & Compartment & & $\begin{array}{c}\text { Compartment } \\
\text { Compartment }\end{array}$ \\
2 & $54.7 \%$ & $45.5 \%$ & & $58.1 \%$ & $45.8 \%$ \\
3 & $38.2 \%$ & $33.8 \%$ & & $60.0 \%$ & $60.0 \%$ \\
4 & $61.9 \%$ & $67.8 \%$ & & $60.8 \%$ & $73.1 \%$ \\
5 & $42.7 \%$ & $39.0 \%$ & & $50.1 \%$ & $37.0 \%$ \\
6 & $45.1 \%$ & $33.6 \%$ & & $48.8 \%$ & $32.2 \%$ \\
7 & $55.2 \%$ & $56.7 \%$ & & $65.9 \%$ & $68.7 \%$ \\
8 & $16.2 \%$ & $21.8 \%$ & & $31.6 \%$ & $24.2 \%$ \\
9 & $39.5 \%$ & $59.2 \%$ & & $58.1 \%$ & $60.4 \%$ \\
10 & $21.9 \%$ & $20.3 \%$ & & $30.2 \%$ & $29.7 \%$ \\
11 & $55.9 \%$ & $63.5 \%$ & & $52.5 \%$ & $59.1 \%$ \\
12 & $21.9 \%$ & $34.5 \%$ & & $35.3 \%$ & $33.5 \%$ \\
Average & $42.1 \%$ & $42.9 \%$ & & $49.3 \%$ & $46.9 \%$ \\
$t$ test & 0.00000004 & 0.0000002 & 0.000000006 & 0.0000002 \\
\hline
\end{tabular}

Percent atrophy of the superior (sup) and inferior (inf) compartments compared with the normal, uninvolved lateral rectus. Larger numbers represent a greater atrophic change. The $t$ tests are statistical comparisons with normal controls with superior compartment maximum cross section $=17.0 \pm 3.0(\mathrm{SD}) \mathrm{mm}^{2}$, inferior compartmentmaximumcross section $=18.2 \pm 3.0 \mathrm{~mm}^{2}$, superior compartment posterior volume $=52.7 \pm 11.4 \mathrm{~mm}^{3}$, and inferior compartment posterior volume $=60.1 \pm 13.2 \mathrm{~mm}^{3}$. 


\section{TABLE 2}

Lateral Rectus Size in Superior Compartment Palsy

\begin{tabular}{|c|c|c|c|c|}
\hline \multicolumn{5}{|c|}{$\begin{array}{l}\text { Asymmetric Lateral Rectus Palsy Percent Atrophy } \\
\text { (Compared With Uninvolved) }\end{array}$} \\
\hline \multirow[b]{3}{*}{ Patient } & \multicolumn{2}{|c|}{ Maximum Cross Section $\left(\mathrm{mm}^{2}\right)$} & \multicolumn{2}{|c|}{ Posterior Volume $\left(\mathrm{mm}^{\mathbf{3}}\right)$} \\
\hline & Sup & Inf & Sup & Inf \\
\hline & Compartment & Compartment & Compartment & Compartment \\
\hline 13 & $73.4 \%$ & $37.6 \%$ & $77.4 \%$ & $52.4 \%$ \\
\hline 14 & $26.3 \%$ & $18.3 \%$ & $45.1 \%$ & $16.2 \%$ \\
\hline 15 & $43.0 \%$ & $17.9 \%$ & $47.3 \%$ & $25.2 \%$ \\
\hline 16 & $24.2 \%$ & $-4.5 \%$ & $32.8 \%$ & $4.2 \%$ \\
\hline 17 & $70.0 \%$ & $50.0 \%$ & $68.7 \%$ & $63.6 \%$ \\
\hline 18 & $42.1 \%$ & $20.9 \%$ & $51.2 \%$ & $27.8 \%$ \\
\hline Average & $46.5 \%$ & $23.4 \%$ & $53.8 \%$ & $31.6 \%$ \\
\hline$t$ test & 0.000006 & 0.34 & 0.000008 & 0.22 \\
\hline
\end{tabular}

Percent atrophy of the superior (sup) and inferior (inf) compartments compared with the normal, uninvolved lateral rectus. Larger numbers represent a greater atrophic change. Patient 16, who underwent both resection and plication of the paretic lateral rectus in separate surgeries prior to imaging, had a larger inferior compartment maximum cross-sectional area on the paretic side than the uninvolved side. The $t$ tests are statistical comparisons with normal controls with superior compartment maximum cross section $=17.0 \pm 3.0$ (SD)mm ${ }^{2}$, inferior compartment maximum cross section $=18.2 \pm 3.0 \mathrm{~mm}^{2}$, superior compartment posterior volume $=52.7 \pm 11.4 \mathrm{~mm}^{3}$, and inferior compartment posterior volume $=60.1 \pm$ $13.2 \mathrm{~mm}^{3}$. 


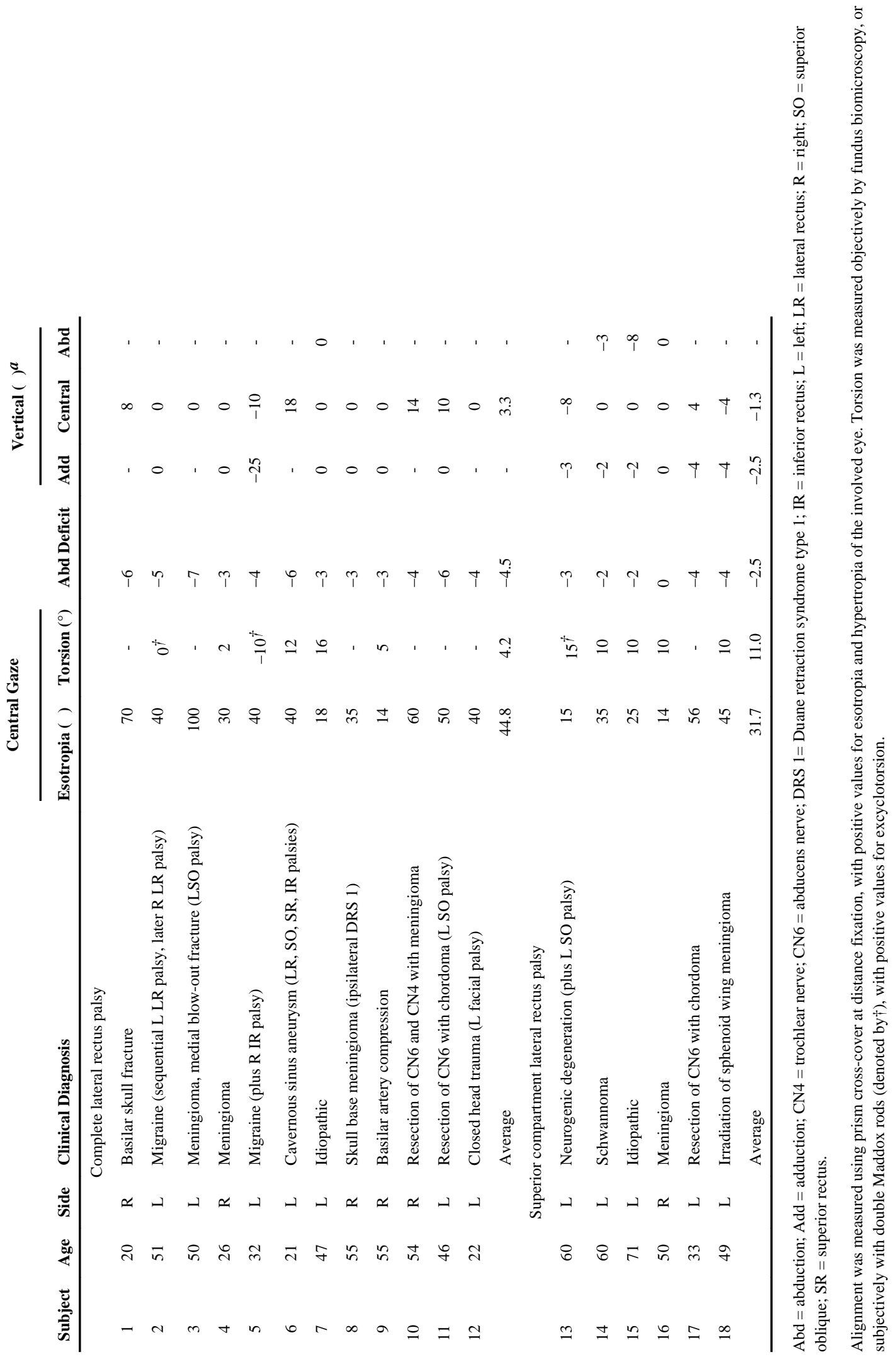


